\title{
An Investigation on M-Polar Fuzzy Saturation Graph and Its Application
}

TANMOY MAHAPATRA ( $\nabla$ tmahapatrapmath@gmail.com )

Vidyasagar University https://orcid.org/0000-0003-2404-1555

Madhumangal Pal

Vidyasagar University

\section{Research Article}

Keywords: m-polar fuzzy graph, a-vertex count of m-polar fuzzy graph, $\beta$-vertex count of m-polar fuzzy graph, saturation in m-polar fuzzy graph.

Posted Date: November 9th, 2021

DOl: https://doi.org/10.21203/rs.3.rs-887604/v1

License: (1) This work is licensed under a Creative Commons Attribution 4.0 International License.

Read Full License 


\title{
An investigation on $m$-polar fuzzy saturation graph and its application
}

\author{
Tanmoy Mahapatra and Madhumangal Pal \\ Department of Applied Mathematics with Oceanology and Computer Programming, \\ Vidyasagar University, Midnapore-721102, India. \\ email: tmahapatrapmath@gmail.com, mmpalvu@gmail.com
}

\begin{abstract}
The saturation graph is a well-defined topic. But, saturation in a fuzzy graph was defined recently and investigated many properties. In a fuzzy saturation graph, only one saturation is considered for every vertex. In a $m$-polar fuzzy graph ( $m$ PFG), each vertex and edge has a $m$ number of membership values. So, defining saturation for $m \mathrm{PFG}$ is not easy and needs some new ideas. By considering $m$ saturation for each component out of $m$ components of membership values of a vertex, we defined saturation in $m$ PFG. $\alpha$-saturation as well as $\beta$-saturation in $m \mathrm{PFG}$ is introduced here. Many interesting properties of it are also presented. $\alpha$-vertex count and $\beta$ vertex count in $m \mathrm{PFG}$ are also studied and the upper bound on some well known $m \mathrm{PFG}$ is also found here. Finally, a real-life application using saturation in $m \mathrm{PFG}$ is also presented.
\end{abstract}

Keywords: m-polar fuzzy graph, $\alpha$-vertex count of m-polar fuzzy graph, $\beta$-vertex count of m-polar fuzzy graph, saturation in m-polar fuzzy graph.

\section{Introduction}

\subsection{Research background and Related works}

Real-world conditions can suitably be stated through a model which consists of nodes set along with lines connecting particular pairs of nodes. Due to graph theory, we present the current interlink between the network or system. It has become conventional to preserve graph theory in different situations such as computer networks, electric networks, etc.

Zadeh [24], in 1965, identified the phenomena of doubtfulness as well as the ambiguity of the real-life situation and brought in a fuzzy set which shifted faces of technology and science. Zhang $[22,23]$ explained the fuzzy set idea as well as presented the possibility of bipolar fuzzy 
sets. Next, Chen et al. [4] presented the thought of fuzzy sets in $m$-dimension as speculation of bipolar fuzzy sets. First, Kafmann [10] presented the fuzzy graph concept utilizing Zadeh's fuzzy relation. After that Rosenfeld [20] supplied the possibility of nodes, edges along with several hypothetical ideas like paths, connectedness, cycle, etc., in fuzziness. Different concepts and definitions are presented thereafter on fuzzy graphs [15, 17, 21]. Nair and Cheng presented fuzzy cliques in fuzzy graphs [18]. Mathew et al. also worked on different properties on fuzzy graphs $[15,17,21]$. Chen et al. [4] first presented a $m$-polar fuzzy graph ( $m$ PFG). Later on, Ghorai and Pal discussed several properties of $m \mathrm{PFG}[6,7,8,9,19]$. Next, Mandal et al. studied different types of arcs on $m$ PFG [13]. Akram and Adeel have also deeded on $m$ PFGs and line graphs [1]. Akram et al. concentrated a few properties of edge on $m$ PFG [2]. Next, Mahapatra and Pal presented fuzzy colouring of $m \mathrm{PFG}$ [11] and recently, Mahapatra et al. studied fuzzy fractional colouring on fuzzy graph [12].

\subsection{Framework of this study}

This paper is structured as follows: Section 2 describe some definitions which are useful in these manuscripts. In section 3, we have discussed the definitions of the strong vertex as well as SE count, $\alpha$-vertex as well as $\alpha$-edge count, $\beta$-vertex as well as $\beta$-edge count of $m \mathrm{PFG}$ and give the lower and upper bound of them in an $m \mathrm{PFG}$. In section 3.1, we investigate vertex as well as edge counts of some well-known $m$ PFG. In section 4 , we introduced saturation in $m \mathrm{PFG}$ with the help of $\alpha$-saturation and $\beta$-saturation. Section 5 describe algorithms to find $\alpha$-saturated as well as $\beta$-saturated and saturation in $m \mathrm{PFG}$. A real-life application based on the allocation problem has been solved using saturation in $m \mathrm{PFG}$, given in Section 6. Lastly, the conclusion has been given in Sections 7 .

\section{Notations and symbols}

In this portion, we reform some of the significant as well as useful notations which are used in the whole paper for development of the theories. The abbreviation form and their meanings are given in Table 1.2.

\begin{tabular}{|c|c|}
\hline Full name & Abbreviation Form \\
\hline Fuzzy graph & FG \\
\hline$m$-polar fuzzy graph & $m$ PFG \\
\hline Underlying crisp graph & UCG \\
\hline Membership value & MV \\
\hline$m$-polar fuzzy set & $m$ PFS \\
\hline Strength of connectedness & SC \\
\hline Strong edge & SE \\
\hline Maximal spanning tree & MST \\
\hline
\end{tabular}

Table 1.2: Abbreviation form of some terms 


\section{Preliminaries}

Here, we briefly call again some basic as well as useful definitions of graphs, $m$ PFG and related terms connecting with it. Suppose $G=(V, E)$ be a graph, where $V$ (non-null set) is indicated as a node-set as well as $E$ is indicated as edge-set. If a node is separate from all edges, then the node is said to be an isolated node. Otherwise, it is called a non-isolated node.

Definition 2.1. [20] A $F G G=(V, \sigma, \mu)$ having $U C G G^{*}$ in which $\sigma: V \rightarrow[0,1]$ as well as $\mu:\left(\widetilde{V^{2}}\right) \rightarrow[0,1]$ is a fuzzy set in $V$ as well as $\widetilde{V^{2}}$ respectively and which obey the following rule $\mu(a, d) \leq\{\sigma(a) \wedge \sigma(d)\}, \forall(a, d) \in \widetilde{V^{2}}$ as well as $\mu(a, d)=0, \forall(a, d) \in\left(\widetilde{V^{2}}-E\right) . \quad \sigma(a)$ as well as $\mu(a, d)$ indicates the node $a$ and edge $(a, d) M V$.

Throughout this article $[0,1]^{m}$ will be a partial order set having component wise order $\leq$, $m \in N$ and " $\leq$ " stands for $x \leq z \Leftrightarrow \forall i=1,2 \ldots m, p_{i}(x) \leq p_{i}(z), x, z$ are taken from $[0,1]^{m}$ as well as $p_{i}:[0,1]^{m} \rightarrow[0,1]$ represents of $i^{t h}$ components of projection mapping.

Definition 2.2. [4] An mPFS $A$ on $X$ is a mapping $A: X \rightarrow[0,1]^{m}$.

Definition 2.3. [4] Let $A$ be an mPFS. Then $h(A)$ denotes height of $A$ and it is given by

$$
\left(\sup _{x \in A} p_{1} \circ A(x), \sup _{x \in A} p_{2} \circ A(x), \ldots, \sup _{x \in A} p_{m} \circ A(x)\right)
$$

Definition 2.4. [15] The support of an $m P F S A$ is given as $\operatorname{supp}(A)=\left\{c \in A: p_{i} \circ B(c)>0\right\}$, $i=1,2, \ldots, m$, indicated by $\operatorname{supp}(A)$, where $B: A \rightarrow[0,1]^{m}$ is a mapping.

Clearly, $\operatorname{supp}(A)=\phi$ iff $A=\phi$ and $\operatorname{supp}(A) \neq \phi$ iff $A \neq \phi$. Therefore, $A$ and $\operatorname{supp}(A)$ are equivalent in $m \mathrm{PFS}$.

Definition 2.5. [7] An mPFG $G=(V, A, B)$ having $U C G G^{*}=(V, E)$, where $A: V \rightarrow[0,1]^{m}$ as well as $B: \widetilde{V \times V} \rightarrow[0,1]^{m}$ represents an $m P F S$ of $V$ as well as $\widehat{V \times V}$ respectively and which obey the rule such that $\forall i=1,2,3, \ldots, m, p_{i} \circ B(a, c) \leq\left\{p_{i} \circ A(a) \wedge p_{i} \circ A(c)\right\} \forall(a, c) \in \widetilde{V \times V}$ as well as $B(a, c)=\mathbf{0} \forall(a, c) \in(\widehat{V \times V}-E) . p_{i} \circ A(a)$ and $p_{i} \circ B(a, c)$ indicates $i^{\text {th }}$ component of membership function of node $a$ and edge $(a, c)$ of $m P F G$.

Definition 2.6. [6] An mPFG $G=(V, \sigma, \mu)$ is entitled as complete whenever $p_{i} \circ \mu(a, c)=$ $\left\{p_{i} \circ \sigma(a) \wedge p_{i} \circ \sigma(c)\right\}, \forall a, c \in V, \quad i=1,2, \ldots, m$.

Definition 2.7. [7] An mPFG $G=(V, \sigma, \mu)$ is entitled as strong whenever

$$
p_{i} \circ \mu(a, c)=\left\{p_{i} \circ \sigma(a) \wedge p_{i} \circ \sigma(c)\right\},
$$

$\forall(a, c) \in E, i=1,2, \ldots, m$

Definition 2.8. [9] Suppose $G=(V, \sigma, \mu)$ as well as $G^{\prime}=\left(V^{\prime}, \sigma^{\prime}, \mu^{\prime}\right)$ be two mPFGs. If there exists a mapping $\phi: G \rightarrow G^{\prime}$ such that for each $i=1,2, \ldots, m$ 
(i) $p_{i} \circ \sigma(a)=p_{i} \circ \sigma^{\prime}(\phi(a)), \forall a \in V$.

(ii) $p_{i} \circ \mu(a, c)=p_{i} \circ \mu^{\prime}(\phi(a), \phi(c)), \forall(a, c) \in \widetilde{V \times V}$.

Then $G$ as well as $G^{\prime}$ are called isomorphic. We write it as $G \cong G^{\prime}$.

Definition 2.9. [3] Let $G=(V, \sigma, \mu)$ be an $m P F G$ having $U C G G^{*}=(V, E)$. Let $H=$ $\left(V^{\prime}, \sigma^{\prime}, \mu^{\prime}\right)$ be a subgraph of $G$ having $U C G H^{*}=\left(V^{\prime}, E^{\prime}\right)$. Then $H$ is called mPF cycle if $\left(\operatorname{supp}\left(V^{\prime}\right), \operatorname{supp}\left(E^{\prime}\right)\right)$ is a cycle and there does not exists unique $(x, y) \in E^{\prime}$ (edge set of $\left.H\right)$ such that $p_{i} \circ \mu(x, z)=\inf \left\{p_{i} \circ \mu(a, c):(a, c) \in E^{\prime}\right\}$, for $i=1,2, \ldots, m$.

Definition 2.10. [13] Suppose $G=(V, \sigma, \mu)$ be an $m P F G$ as well as $P: a_{1}, a_{2}, \ldots, a_{k}$ be a path in $G$. Then $S(P)$ denotes the strength of the path $P$ which is given by $S(P)=\left(\min _{1 \leq i<j \leq k} p_{1} \circ\right.$ $\left.\mu\left(a_{i}, a_{j}\right), \min _{1 \leq i<j \leq k} p_{2} \circ \mu\left(a_{i}, a_{j}\right), \ldots, \min _{1 \leq i<j \leq k} p_{m} \circ \mu\left(a_{i}, a_{j}\right)\right)=\left(\mu_{1}^{n}\left(a_{i}, a_{j}\right), \mu_{2}^{n}\left(a_{i}, a_{j}\right), \ldots, \mu_{m}^{n}\left(a_{i}, a_{j}\right)\right)$.

The $S C$ of the path in between $\bar{a}_{1}$ and $a_{k}$ is given in the following way: $C O N N_{G}\left(a_{1}, a_{k}\right)=$ $\left(p_{1} \circ \mu\left(a_{i}, a_{j}\right)^{\infty}, p_{2} \circ \mu\left(a_{i}, a_{j}\right)^{\infty}, \ldots, p_{m} \circ \mu\left(a_{i}, a_{j}\right)^{\infty}\right)$, where $\left(p_{i} \circ \mu(a, b)^{\infty}\right)=\max _{n \in N}\left(\mu_{i}^{n}(a, b)\right)$.

Definition 2.11. [13] Suppose $G=(V, \alpha, \mu)$ is an $m P F G$ as well as $(x, z)$ be an edge in $G$. If $\forall i=1,2, \ldots, m, p_{i} \circ \mu(x, z)>p_{i} \circ C O N N_{G-(x, z)}(x, z), p_{i} \circ \mu(x, z)=p_{i} \circ C O N N_{G-(x, z)}(x, z)$, $p_{i} \circ \mu(x, z)<p_{i} \circ C O N N_{G-(x, z)}(x, z)$ then $(x, z)$ is called $\alpha$-strong, $\beta$-strong, $\delta$-SE respectively.

Definition 2.12. [14] An mPFG $G=(V, \sigma, \mu)$ is said to be mPF tree if there exists a spanning $m P F$ subgraph $H=\left(V, \sigma^{\prime}, \mu^{\prime}\right)$ which is an $m P F$ tree and $p_{i} \circ \mu^{\prime}(a, c)=0$ means $p_{i} \circ$ $C O N N_{H}(a, c)>p_{i} \circ \mu^{\prime}(a, c)$, for $i=1,2, \ldots, m$.

Definition 2.13. [14] Suppose $G=(V, \alpha, \mu)$ is an mPFG. An arc $(a, c)$ is said to be mPF bridge if deletion of its decreases the $S C$ between some other pair of vertices of $G$.

In this article $G^{*}=(V, E)$ stands for the UCG of an $m \mathrm{PFG} G$.

\section{Vertex as well as edge saturation counts of $m$ PFG}

In this section, we introduced vertex as well as edge saturation counts in $m$ PFG and discussed various useful properties of them. Vertex saturation count of an $m$ PFG gives the dimension of the mean strong degree of $m \mathrm{PFG}$ and edge saturation count indicates the portion of SEs of $m$ PFG. Here, we consider $\sigma(u)=\mathbf{1}=(1,1, \ldots, 1)$, for all $u \in V$, where $G=(V, \sigma, \mu)$.

Definition 3.1. Suppose $G=(V, \sigma, \mu)$ be an $m P F G$ having $U C G G^{*}=(V, E)$. Then strong vertex count of $G$ is indicated by $S_{V}(G)$ and given by

$$
\begin{aligned}
p_{i} \circ S_{V}(G) & =\frac{\text { number of } S E \text { of } G}{|V|} \\
& =\frac{\text { number of } \alpha \text { or } \beta-S E \text { of } G}{|V|}
\end{aligned}
$$

$\forall i=1,2, \ldots, m$ and $S E$ count of $G$ is indicated by $S_{E}(G)$ as well as given by 
$\forall i=1,2, \ldots, m$.

Definition 3.2. Suppose $G=(V, \sigma, \mu)$ be an $m P F G$ having $U C G G^{*}=(V, E)$. Then $\alpha$-vertex count of $G$ is indicated by $\alpha_{V}(G)$ as well as given by

$$
p_{i} \circ \alpha_{V}(G)=\frac{\text { number of } \alpha-S E \text { of } G}{|V|}
$$

$\forall i=1,2, \ldots, m$ and $\alpha-S E$ count of $G$ is indicated by $\alpha_{E}(G)$ as well as given by

$$
p_{i} \circ \alpha_{E}(G)=\frac{\text { number of } \alpha-S E \text { of } G}{|E|}
$$

$\forall i=1,2, \ldots, m$.

Definition 3.3. Suppose $G=(V, \sigma, \mu)$ is an mPFG having $U C G G^{*}=(V, E)$. Then $\beta$-vertex count of $G$ is indicated by $\alpha_{V}(G)$ as well as given by

$$
p_{i} \circ \beta_{V}(G)=\frac{\text { number of } \beta-S E \text { of } G}{|V|}
$$

$\forall i=1,2, \ldots, m$ and the $\beta$-SE count of $G$ is indicated by $\beta_{E}(G)$ and given by

$$
p_{i} \circ \beta_{E}(G)=\frac{\text { number of } \beta-S E \text { of } G}{|E|}
$$

$\forall i=1,2, \ldots, m$.

Example 1. Here, we consider an $3 P F G$ to depicted the above definitions. Here, we consider $\sigma(x)=(1,1,1)$, for all $x \in V$.

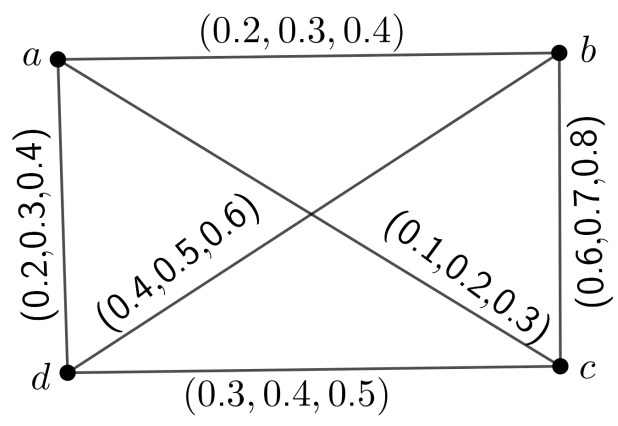

Figure 1: 3PFG G

Here, the classified edges are given in tabular form.

\begin{tabular}{|c|r|}
\hline Edge & Classification \\
\hline$(a, b)$ & $\beta$-strong \\
\hline$(b, c)$ & $\alpha$-strong \\
\hline$(a, d)$ & $\beta$-strong \\
\hline$(b, d)$ & $\alpha$-strong \\
\hline$(a, c)$ & $\delta$-strong \\
\hline$(d, c)$ & $\delta$-strong \\
\hline
\end{tabular}


$\alpha$-vertex count of $G$ is

$$
p_{i} \circ \alpha_{V}(G)=\frac{2}{4}=\frac{1}{2}, \text { for } i=1,2,3
$$

and $\alpha$-edge count of $G$ is

$$
p_{i} \circ \alpha_{E}(G)=\frac{2}{6}=\frac{1}{3}, \text { for } i=1,2,3 \text {. }
$$

$\beta$-vertex count of $G$ is

$$
p_{i} \circ \beta_{V}(G)=\frac{2}{4}=\frac{1}{2}, \text { for } i=1,2,3
$$

and $\beta$-edge count of $G$ is

$$
p_{i} \circ \beta_{E}(G)=\frac{2}{6}=\frac{1}{3}, \text { for } i=1,2,3
$$

Strong-vertex count of $G$ is

$$
p_{i} \circ S_{V}(G)=\frac{4}{4}=1,, \text { for } i=1,2,3
$$

and Strong-edge count of $G$ is

$$
p_{i} \circ S_{E}(G)=\frac{4}{6}=\frac{2}{3}, \text { for } i=1,2,3 .
$$

Since every edges in an $m \mathrm{PF}$ tree is $\alpha$-strong by the Theorem 3.18 of [13] therefore $p_{i} \circ \alpha_{V}(G)=$ $\frac{n-1}{n}$ and $p_{i} \circ \alpha_{E}(G)=\frac{n-1}{n-1}=1$, where $n=$ total number of vertex in an $m \mathrm{PF}$ tree $G=(V, \sigma, \mu)$.

Any other $m \mathrm{PFG}$ except the $m \mathrm{PF}$ tree, the count of $\alpha$-strong vertex never exceeds the count of nodes. For a complete $m \mathrm{PFG}$, all possible edges can be made $\beta$-strong by allotting the same MV to the nodes. Then $p_{i} \circ \beta_{V}(G)=\frac{\left(\begin{array}{c}n \\ 2\end{array}\right)}{2}$ and $p_{i} \circ \beta_{E}(G)=\frac{\left(\begin{array}{l}n \\ 2\end{array}\right)}{\left(\begin{array}{c}n \\ 2\end{array}\right)}=1$, for $i=1,2, \ldots, m$.

Depending on the above observation, we can say the following:

Proposition 1. Suppose $G=(V, \sigma, \mu)$ is an $m P F G$ where $|V|=n$. Then

(i) $0 \leq p_{i} \circ \alpha_{V}(G) \leq \frac{n-1}{n}$.

(ii) $0 \leq p_{i} \circ \alpha_{E}(G) \leq 1$.

(iii) $0 \leq p_{i} \circ \beta_{V}(G) \leq \frac{\left(\begin{array}{l}n \\ 2\end{array}\right)}{2}$.

(iv) $0 \leq p_{i} \circ \beta_{E}(G) \leq 1$.

(v) $0 \leq p_{i} \circ S_{V}(G) \leq \frac{\left(\begin{array}{c}n \\ 2\end{array}\right)}{2}$.

(vi) $0 \leq p_{i} \circ S_{E}(G) \leq 1$.

for each $i=1,2, \ldots, m$. 
Proposition 2. Suppose $G=(V, \sigma, \mu)$ is an mPF tree. Then $0 \leq p_{i} \circ \alpha_{V}(G) \leq p_{i} \circ \alpha_{E}(G)$, $\forall i=1,2, \ldots, m$.

Proof. As $G$ is an $m \mathrm{PF}$ tree therefore $p_{i} \circ \alpha_{V}(G)=\frac{n-1}{n}, \forall i=1,2, \ldots, m$ and $p_{i} \circ \alpha_{E}(G)=$ $\frac{n-1}{n-1}=1, \forall i=1,2, \ldots, m$. Hence, $0 \leq p_{i} \circ \alpha_{V}(G) \leq p_{i} \circ \alpha_{E}(G), \forall i=1,2, \ldots, m$.

\subsection{Vertex and edge counts of some well-known $m$ PFG}

In this portion, we talk over saturation counts of $m \mathrm{PFG}$ structures like $m \mathrm{PF}$ cycles, trees, and blocks in $m$ PFG. Some necessary parts for these structures are also obtained.

Theorem 3.1. Suppose $G=(V, \sigma, \mu)$ be an mPFG having $U C G G^{*}=(V, E)$ where $|V|=n$. Then, the following condition are identical:

(i) $G$ be an $m P F$ tree.

(ii) $p_{i} \circ \alpha_{V}(G)=\frac{n-1}{n}$ as well as $p_{i} \circ \alpha_{E}(G)=1, \forall i=1,2, \ldots, m$.

(iii) $n \times p_{i} \circ \alpha_{V}(G)=(n-1) \times p_{i} \circ \alpha_{E}(G), \forall i=1,2, \ldots, m$.

Proof. $(i) \Rightarrow(i i)$ done previously.

$($ ii $) \Rightarrow($ iii $)$

Suppose that $p_{i} \circ \alpha_{V}(G)=\frac{n-1}{n}$ and $p_{i} \circ \alpha_{E}(G)=1, \forall i=1,2, \ldots, m$.

$$
\begin{aligned}
p_{i} \circ \alpha_{V}(G) & =\frac{n-1}{n} \\
\Rightarrow n \times p_{i} \circ \alpha_{V}(G) & =(n-1) \\
\Rightarrow n \times p_{i} \circ \alpha_{V}(G) & =(n-1) \times 1 \\
\Rightarrow n \times p_{i} \circ \alpha_{V}(G) & =(n-1) \times p_{i} \circ \alpha_{E}(G) \quad\left[\text { As } p_{i} \circ \alpha_{E}(G)=1\right]
\end{aligned}
$$

Hence, $n \times p_{i} \circ \alpha_{V}(G)=(n-1) \times p_{i} \circ \alpha_{E}(G), \forall i=1,2, \ldots, m$.

$($ iii $) \Rightarrow(i)$

Suppose that $n \times p_{i} \circ \alpha_{V}(G)=(n-1) \times p_{i} \circ \alpha_{E}(G), \forall i=1,2, \ldots, m$.

Since,

$$
\begin{aligned}
& n \times p_{i} \circ \alpha_{V}(G)=(n-1) \times p_{i} \circ \alpha_{E}(G) \\
& \Rightarrow \frac{p_{i} \circ \alpha_{V}(G)}{p_{i} \circ \alpha_{E}(G)}=\frac{(n-1)}{n} \\
& \Rightarrow \frac{p_{i} \circ \alpha_{V}(G)}{p_{i} \circ \alpha_{E}(G)}=\frac{(n-1)}{n}=p_{i} \circ \alpha_{V}(G)
\end{aligned}
$$

This shows that $p_{i} \circ \alpha_{E}(G)=1$, for each $i=1,2, \ldots, m$.

Hence, $G$ is connected and acyclic only when all edges are $\alpha$-strong and therefore, $G$ is a tree. 
Theorem 3.2. Suppose $G=(V, \sigma, \mu)$ is a connected $m P F G$. $G$ is an $m P F$ tree iff $p_{i} \circ \alpha_{V}(G)=$ $p_{i} \circ S_{V}(G)$ as well as $p_{i} \circ \alpha_{E}(G)=p_{i} \circ S_{E}(G), \forall i=1,2, \ldots, m$.

Proof. Suppose $G$ be a connected $m \mathrm{PFG}$ as well as $m \mathrm{PF}$ tree. Now from Theorem 3.19 of [13], we know that $G$ is free from $\beta$-SEs. Therefore,

$$
p_{i} \circ \beta_{V}(G)=\frac{0}{|V|}=0, \forall i=1,2, \ldots, m
$$

and

$$
p_{i} \circ \beta_{E}(G)=\frac{0}{|E|}=0, \forall i=1,2, \ldots, m .
$$

Therefore,

$$
p_{i} \circ \alpha_{V}(G)=p_{i} \circ S_{V}(G), \forall i=1,2, \ldots, m
$$

and

$$
p_{i} \circ \alpha_{E}(G)=p_{i} \circ S_{E}(G), \forall i=1,2, \ldots, m .
$$

Conversely, let $p_{i} \circ \alpha_{V}(G)=p_{i} \circ S_{V}(G)$ and $p_{i} \circ \alpha_{E}(G)=p_{i} \circ S_{E}(G)$, for $i=1,2, \ldots, m$. If $G$ is acyclic, then $G$ is an $m \mathrm{PF}$ tree. Let $C$ be a cycle in $G$. Hence $C$ must have only $\alpha$-strong as well as $\delta$-SEs only. Again, consider $G$ does not have all $\alpha$-SEs. Therefore, $G$ contains at least one $\delta$-SE. Suppose $e$ is an $\delta$-SE. Then, we remove it from $C$. If a unique MST is found then the condition is done. Otherwise, removing $\delta$-SEs one by one from $C$ until we get a unique MST of $G$.

Theorem 3.3. A connected $m P F G G=(V, \sigma, \mu)$ be an $m P F$ tree iff $p_{i} \circ \alpha_{V}(G)=p_{i} \circ \alpha_{V}(F)$, for each $i=1,2, \ldots, m$, where $F$ is $M S T$ of $G$.

Proof. Suppose $G$ is an $m \mathrm{PF}$ tree. Then $G$ and $F$ are isomorphic. Therefore,

$$
\begin{aligned}
p_{i} \circ \alpha_{V}(G) & =\frac{\text { count of } \alpha-S E \text { of } G}{\text { number of nodes }} \\
& =\frac{\text { count of } \alpha-S E \text { of } F}{\text { number of nodes }} \\
& =p_{i} \circ \alpha_{V}(F)
\end{aligned}
$$

for $\mathrm{i}=1,2, \ldots, \mathrm{m}$.

Now, we consider another case. Suppose, $G$ contains a cycle, say $C$. Then, it is not free from $\delta$-SE. Let $e$ be a $\delta$-SE . If $G-e$ is a tree therefore $G-e$ as well as $F$ are isomorphic. Therefore,

$$
p_{i} \circ \alpha_{V}(G)=p_{i} \circ \alpha_{V}(F), \forall i=1,2, \ldots, m .
$$

If $G-e$ is not a tree, deleting the $\delta$-SEs in $G-e$ in similar manner to obtain a MST $F$ of $G$ such that $p_{i} \circ \alpha_{V}(G)=p_{i} \circ \alpha_{V}(F)$, for each $i=1,2, \ldots, m$.

Conversely, let $p_{i} \circ \alpha_{V}(G)=p_{i} \circ \alpha_{V}(F), \forall i=1,2, \ldots, m$, where $F$ is the corresponding MST of $G$. We have to show that $G$ is an $m \mathrm{PF}$ tree. Suppose $G$ is not an $m \mathrm{PF}$ tree, then it must have one $\beta$-SE, say $(a, b)$. Let $c-d$ be another path $P$ in $G$ for which $p_{i} \circ \mu(c, d) \geq p_{i} \circ \mu(a, b)$, 
$\forall i=1,2, \ldots, m$ and $\forall(c, d) \in P$. Now, the join of $P$ as well as $(a, b)$ creates a cycle in $G$. Let $k$ be the count of $\alpha$-SEs which are incident at $a$. To find $F$, remove $(a, b)$ from $G$, which has minimum weight in $C$. Then the count of $\alpha$-SEs connected to $c$ in $F$ is $k+1$. Suppose the remaining counts of $\alpha$-SEs is $k_{1}$. Hence, $p_{i} \circ \alpha_{V}(G)=\frac{k+1}{|V|}$ as well as $p_{i} \circ \alpha_{V}(F)=\frac{k+k_{1}+1}{|V|}$, $\forall i=1,2, \ldots, m$, a contrast. Therefore, the theorem.

\section{Saturation in $m$-polar fuzzy graph}

Here, saturation in terms of a node as well as edge counts is presented. In this section, we also studied some of the interesting facts of it. We also studied saturated blocks in $m$ PFG.

Definition 4.1. Suppose $G$ is an $m P F G$. Then $G$ is called $\alpha$-saturate if it must have one $\alpha$-SEs incident with each node of $G$. $G$ is said to be $\beta$-strong saturate if it must have one $\beta$-SE incident with each nodes of $G$.

Definition 4.2. Suppose $G$ is an mPFG. $G$ is called a saturate graph if at least one $\alpha$-SE as well as $\beta$-SEs incident with every vertex of $G$. Otherwise $G$ is called an unsaturated $m P F G$.

Example 2. To illustrate the above definition we consider a $3 P F G G$ displayed in Fig. 2 whose all vertices having membership value $(1,1,1)$.

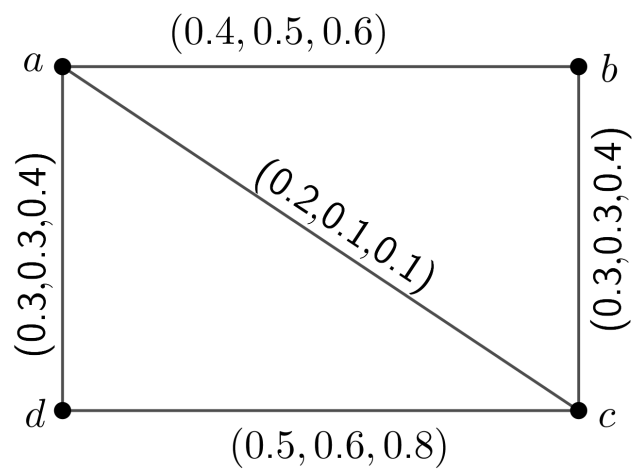

Figure 2: 3PFG $G$ having four vertices

Here, we see that the edges $(a, b),(c, d)$ are $\alpha$-SEs and $(c, b),(a, d)$ are $\beta$-SEs. The edges $(a, c)$ are $\delta$-SE. Each vertex is connected with $\alpha$-SE as well as $\beta$-SE. Therefore, $G$ be a saturated $3 P F G$.

Theorem 4.1. Suppose $G=(V, \sigma, \mu)$ as well as $G^{\prime}=\left(V^{\prime}, \sigma^{\prime}, \mu^{\prime}\right)$ are two isomorphic mPFGs. If $G$ is saturated, $G^{\prime}$ is also saturated.

Proof. Let $\phi: G \rightarrow G^{\prime}$ be the isomorphism between two mPFGs. Therefore, we have $\forall i=1,2, \ldots, m$

(i) $p_{i} \circ \sigma(a)=p_{i} \circ \sigma^{\prime}(\phi(a)), \forall a \in V$. 
(ii) $p_{i} \circ \mu(a, c)=p_{i} \circ \mu^{\prime}(\phi(a), \phi(c)), \forall(a, c) \in \widetilde{V \times V}$.

To show $G^{\prime}$ is saturated, we have to show that each node is connected with at least one $\alpha$-SE as well as $\beta$-SEs. Let $w^{\prime} \in V^{\prime}$. Then there must be a node, say $w$, in $G$ for which $\phi(w)=w^{\prime}$. Since $G$ is saturated, therefore $w$ is an incident with at least one $\alpha$-SEs as well as $\beta$-SEs. Since, $G$ and $G^{\prime}$ are isomorphic to each other. Therefore, $w^{\prime}$ is also incident to at least one $\alpha$-SEs and $\beta$-SEs. Hence, $G^{\prime}$ is also saturated.

Let $G$ be an $m$ PFG having UCG $G^{*}=(V, E)$ where $|V|=k$. We define a finite sequence $\alpha_{S}(G)=\left(n_{1}, n_{2}, \ldots, n_{k}\right)$ called $\alpha$-strong sequence where, $n_{j}=$ count of $\alpha$-SEs connect at node $v_{j}$. We define a finite sequence $\beta_{S}(G)=\left(n_{1}, n_{2}, \ldots, n_{k}\right)$ called $\beta$-strong sequence where, $n_{j}=$ count of $\beta$-SEs connect at node $v_{j}$. Since, the count of SEs of $G=($ the count of $\alpha$-SEs of $G+$ the count of $\beta$-SEs of $G$ ) therefore,

$$
\sum_{n_{j} \in \alpha_{S}(G)} n_{j}+\sum_{n_{j} \in \beta_{S}(G)} n_{j}=\sum_{n_{j} \in S_{S}(G)} n_{j}
$$

Theorem 4.2. Suppose $G=(V, \sigma, \mu)$ is an $m P F G$ having $U C G G^{*}=(V, E)$ where $|V|=k$. Then $G$ is $\alpha$-saturated iff $\sum_{n_{j} \in \alpha_{S}(G)} n_{j} \geq k$.

Proof. Suppose $G$ be an $\alpha$-saturated $m \mathrm{PFG}$. Therefore, at least one $\alpha$-SEs incident with each vertex of $G$. Thus

$$
\begin{aligned}
& \sum_{n_{j} \in \alpha_{S}(G)} n_{j} \geq 1+1+\ldots+1 \\
\Rightarrow & \sum_{n_{j} \in \alpha_{S}(G)} n_{j} \geq k
\end{aligned}
$$

Conversely, let $\sum_{n_{j} \in \alpha_{S}(G)} n_{j} \geq k$. Then, all $k$ nodes of $G$ are connected with at least one $\alpha$-SEs. Therefore, $G$ is $\alpha$-saturated $m$ PFG.

Theorem 4.3. Suppose $G=(V, \sigma, \mu)$ is an $m P F G$ having $U C G G^{*}=(V, E)$ where $|V|=k$. Then $G$ is $\beta$-saturated iff $\sum_{n_{j} \in \beta_{S}(G)} n_{j} \geq k$.

Proof. Similar to the above theorem.

Theorem 4.4. Suppose $G=(V, \sigma, \mu)$ is an mPFG having $U C G G^{*}=(V, E)$ where $|V|=k$. If $G$ is $\beta$-saturated then $\sum_{n_{j} \in S_{S}(G)} n_{j} \geq 2 k$.

Proof. Let $G$ be saturated. Therefore, each node of $G$ is connected with at least one $\alpha$-SE as well as one $\beta$-SE. Thus, $\sum_{n_{j} \in S_{S}(G)} n_{j} \geq 2 k$. 
Theorem 4.5. Suppose $G=(V, \sigma, \mu)$ is an $m P F G$ having $U C G G^{*}=(V, E)$ where $|V|=n$. If,

(i) $p_{i} \circ \alpha_{V}(G) \geq 0.5$ if $\alpha$-saturated.

(ii) $p_{i} \circ \beta_{V}(G) \geq 0.5$ if $\beta$-saturated.

(iii) $p_{i} \circ S_{V}(G) \geq 1$ if saturated.

$\forall i=1,2, \ldots, m$.

Proof. i) Let $G$ is $\alpha$-saturated then every vertex of $G$ is incident with at least one $\alpha$-SEs. Therefore, $G$ must have $\frac{n}{2}, \alpha$-SEs. Therefore, $p_{i} \circ \alpha_{V}(G) \geq \frac{\frac{n}{2}}{n}=0.5$.

ii) Similar to the above.

iii) Let $G$ be saturated. Therefore, each node of $G$ is connected with at least one $\alpha$-SE as well as $\beta$-SEs. Since, the count of SEs of $G=$ (the count of $\alpha$-SEs of $G+$ the count of $\beta$-SEs of $G) \geq \frac{n}{2}+\frac{n}{2}=n$. Hence, $p_{i} \circ S_{V}(G) \geq \frac{n}{n}=1$.

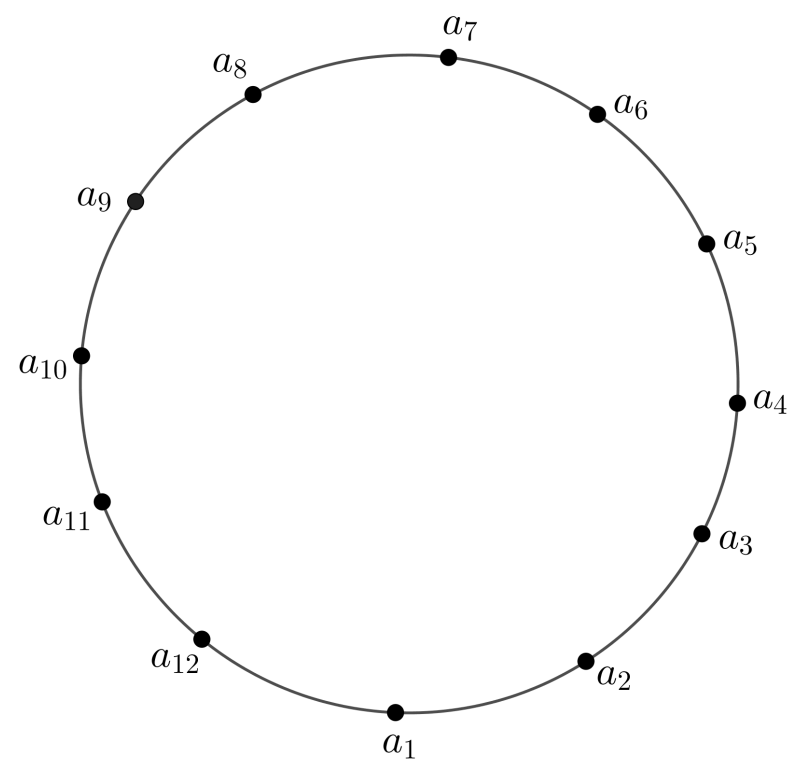

Figure 3: 3PFG $G$ having even number of vertices

In the Fig. 3, all the vertices have membership value $(1,1,1)$, that is $\sigma\left(a_{j}\right)=(1,1,1)$, for $j=$ $1,2, \ldots, 12$. The edges membership value is $\mu\left(a_{j}, a_{k}\right)=(0.6,0.6,0.6)$, where $1 \leq j<k \leq 12$ and $j$ is odd and $k$ is even. The edges membership value is $\mu\left(a_{j}, a_{k}\right)=(0.4,0.4,0.4)$, where $1<j<k<12$ and $j$ is even and $k$ is odd. The edge membership value between $a_{1}$ and $a_{12}$ is $(0.4,0.4,0.4)$.

In the Fig. 3, we see that all the edges having membership value $(0.6,0.6,0.6)$ are $\alpha$-strong and the edges having membership value $(0.4,0.4,0.4)$ are $\beta$-strong. Therefore, Fig. 3 is saturated. 


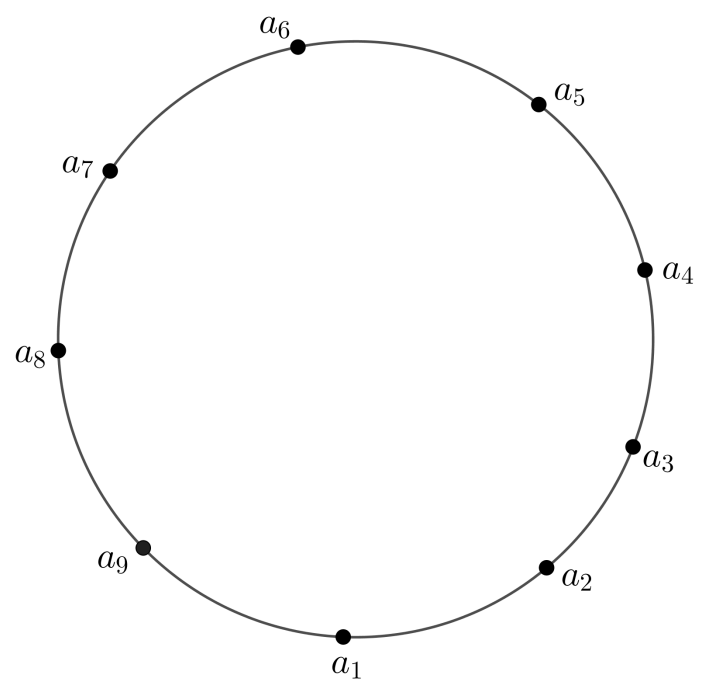

Figure 4: $3 \mathrm{PFG} G$ having odd number of vertices

In the Fig. 4, all the vertices have membership value $(1,1,1)$, that is $\sigma\left(a_{j}\right)=(1,1,1)$, for $j=$ $1,2, \ldots, 9$. The edges membership value is $\mu\left(a_{j}, a_{k}\right)=(0.5,0.5,0.5)$, where $1 \leq j<k \leq 9$ and $j$ is odd and $k$ is even. The edges membership value is $\mu\left(a_{j}, a_{k}\right)=(0.7,0.7,0.7)$, where $1<j<k<9$ and $j$ is even and $k$ is odd. The edge membership value between $a_{1}$ and $a_{9}$ is $(0.5,0.5,0.5)$.

In the Fig. 4 , we see that all the edges having membership value $(0.7,0.7,0.7)$ are $\alpha$-strong and the edges having membership value $(0.5,0.5,0.5)$ are $\beta$-strong. Therefore, Fig. 4 is unsaturated as the vertex $a_{1}$ connected with both the $\beta$-SEs.

One simple observation of the above discussion is that Fig. 3 has an even number of vertices while in Fig. 4 has an odd number of vertices. Thus we have the following theorem.

Theorem 4.6. Suppose $C_{n}$ be an mPF cycle. It is saturated iff the following two hold:

(i) $n=2 t, t$ is a positive integer.

(ii) $\alpha$-SE as well as $\beta$-SEs occur alternatively on $C_{n}$.

Proof. Suppose $C_{n}$ is an $m \mathrm{PF}$ cycle. Therefore, it is free from $\delta$-SEs. All arcs that occur on $C_{n}$ are $\alpha$-SE or $\beta$-SE. Let us assume that $C_{n}$ be saturated. Therefore, each node is connected with at least one $\alpha$-SE and one $\beta$-SEs. Hence, count of $\alpha$-SEs $=t=$ count of $\beta$-SEs. Therefore, $n=2 t$. Again, every node connected with both $\alpha$-SE as well as $\beta$-SEs happen if they occur alternatively on $C_{n}$.

Conversely, let $C_{n}$ is a fuzzy cycle with an even number of nodes in which each node is connected with both $\alpha$-SE as well as $\beta$-SEs alternatively. Therefore, each node is connected with precisely one $\alpha$-SE as well as $\beta$-SEs. Hence, $C_{n}$ be a saturated fuzzy cycle.

Theorem 4.7. Suppose $G=(V, \sigma, \mu)$ be an $m P F$ cycle. If $G$ is saturated, it must be a block.

Proof. Since $G$ is saturated, each node is connected with at least one $\alpha$-SE as well as $\beta$ SEs. Again, since $G$ is an $m \mathrm{PF}$ cycle; therefore every node is connected with just two nodes. 
Therefore, every node is incident with precisely one $\alpha$-SE and $\beta$-SEs. Hence, removing any node from $G$ may not decrease SC between other nodes. This shows that $G$ is free from the $m \mathrm{PF}$ cut node; therefore, $G$ is a block.

Theorem 4.8. Let $G=(V, \sigma, \mu)$ is $m P F$ cycle. If $G$ be an mPF blocks, then it is either saturated or $\beta$-saturated.

Proof. Let a block be $G$. We demand that $G$ is free from $\delta$-SEs. If possible, let $e$ be a $\delta$-SE. Then the remaining edges must be $\alpha$-SE, and therefore $G$ contains $n-2$ fuzzy cut nodes, an irrelevance. So, $G$ has no $\delta$-SEs. Thus, $G$ is free from $\delta$-SEs.

If $G$ has only $\alpha$-SE as well as $\beta$-SEs, they appear alternatively; else, the block shape will not be found. If count of $\alpha$-SE $=$ count of $\beta$-SEs $=\frac{n}{2}$, then $G$ be $\alpha$-saturated as well as $\beta$-saturated and therefore it is saturated. If the count of $\alpha$-SEs is less than the count of $\beta$-SEs, then $G$ must be only $\beta$-saturated. For another case, when the count of $\alpha$-SEs is greater than the count of $\beta$-SEs, it will not be true as it does not form a block. If every arc is $\beta$-strong of $G$, it must be $\beta$-saturated. Therefore, the theorem.

Theorem 4.9. A complete $m P F G$ has no $\delta$-arcs.

Proof. Suppose $G=(V, \sigma, \mu)$ is a complete $m$ PFG. Let $G$ has a $\delta$-arcs. Let $(p, q)$ be the $\delta$-arcs. Then we have,

$$
p_{i} \circ \mu(p, q)<p_{i} \circ C O N N_{G-(p, q)} \mu(p, q), \forall i=1,2, \ldots, m .
$$

A stronger path $P$ except the arc $(p, q)$ in $G$ must exist there. Suppose $p_{i} \circ \mu(p, q)=t_{i}$, $i=1,2, \ldots, m$ and the strength of $P$ be $\left(u_{1}, u_{2}, \ldots, u_{m}\right)$. Therefore, we have $t_{i}<u_{i}, \forall i=$ $1,2, \ldots, m$. Suppose $r$ be the first vertex after $u$ in the path $P$. Then, we have

$$
p_{i} \circ \mu(p, r)>t_{i}, \forall i=1,2, \ldots, m
$$

In a similar way, let $s$ is the last vertex before $q$ in the path $P$. Again, we also have

$$
p_{i} \circ \mu(s, q)>t_{i}, \forall i=1,2, \ldots, m
$$

Since, $G$ be complete $m \mathrm{PFG}$, therefore we have $p_{i} \circ \mu(p, q)=\min \left\{p_{i} \circ \sigma(p), p_{i} \circ \sigma(q)\right\}, \forall i=$ $1,2, \ldots, m$ as well as $\forall(p, q) \in E$. Therefore, at least one of $p_{i} \circ \sigma(p)$ or $p_{i} \circ \sigma(q)$ be $t_{i}$, $\forall i=1,2, \ldots, m$.

Therefore, (1) will contradict if $p_{i} \circ \sigma(p)=t_{i}, \forall i=1,2, \ldots, m$ and (2) will contradict if $p_{i} \circ \sigma(q)=t_{i}$, for $i=1,2, \ldots, m$.

Hence, the theorem.

Theorem 4.10. Suppose $G=(V, \sigma, \mu)$ is an $m P F G$. An arc $(a, c)$ be an $m P F$ bridge iff it is $\alpha$-strong.

Proof. Suppose $(a, c)$ be an $m \mathrm{PF}$ bridge. Then we have from the definition of $m \mathrm{PF}$ bridge, $p_{i} \circ C O N N_{G-(a, c)}(a, c)<p_{i} \circ C O N N_{G}(a, c)$, for $i=1,2, \ldots, m$ 
Again, from Theorem 3.11 of [13], we have $p_{i} \circ \mu(a, c)=p_{i} \circ C O N N_{G}(a, c), \forall i=1,2, \ldots, m$

From (1) and (2), we get $p_{i} \circ \mu(a, c)>p_{i} \circ C O N N_{G-(a, c)}(a, c), \forall i=1,2, \ldots, m$. Hence, $(a, c)$ be $\alpha$-SE.

Conversely, suppose $(a, c)$ is an $\alpha$-SE. Then, we have $(a, c)$ is the one and only one strongest path in between $a$ and $c$ and removal of $(a, c)$ will decrease the SC of $a$ and $b$. Therefore, $(a, c)$ is a bridge.

Theorem 4.11. A complete mPFG has at most one $\alpha$-SEs.

Proof. We know that complete $m \mathrm{PFG}$ have at most one $m \mathrm{PF}$ bridge. Again, from Theorem 4.10 , we have an arc $(a, b)$ be an $m \mathrm{PF}$ bridge iff it is $\alpha$-SE. Hence, a complete $m \mathrm{PFG}$ has at most one $\alpha$-SEs.

Proposition 3. Every complete mPFG has at most $\left(\begin{array}{l}n \\ 2\end{array}\right)$ or $\left(\begin{array}{l}n \\ 2\end{array}\right)-1 \beta$-SEs.

Theorem 4.12. If $G$ is a complete $m P F G$ having $n$ vertices, the following inequalities hold.

(i) $0 \leq p_{i} \circ \alpha_{V}(G) \leq \frac{1}{n}$.

(ii) $\frac{n^{2}-n-2}{2 n} \leq p_{i} \circ \beta_{V}(G) \leq \frac{n-1}{2}$.

$\forall i=1,2, \ldots, m$.

Proof. With the help of Theorem 4.11, we have $G$ have at most one $\alpha$-SEs. Hence, we have $p_{i} \circ \alpha_{V}(G) \leq \frac{1}{n}$, for each $i=1,2, \ldots, m$. Again, clearly $p_{i} \circ \alpha_{V}(G) \geq 0$, for each $i=1,2, \ldots, m$. Therefore, $0 \leq p_{i} \circ \alpha_{V}(G) \leq \frac{1}{n}$, for each $i=1,2, \ldots, m$.

Again, from Proposition 3, we know that the minimum number of $\beta$-SEs are $\left(\begin{array}{l}n \\ 2\end{array}\right)-1$. Therefore,

$$
\begin{aligned}
p_{i} \circ \beta_{V}(G) & \geq \frac{n\left(\frac{n-1}{2}\right)-1}{n} \\
& \geq \frac{n^{2}-n-2}{2 n}
\end{aligned}
$$

Thus, $\frac{n^{2}-n-2}{2 n} \leq p_{i} \circ \beta_{V}(G) \leq \frac{n-1}{2}$, for each $i=1,2, \ldots, m$.

Next, we will try to find out the upper limit of $\alpha$-node count for a block in $m$ PFG.

Theorem 4.13. If $G=(V, \sigma, \mu)$ is an $m P F$ blocks, we have $p_{i} \circ \alpha_{V}(G) \leq 0.5, \forall i=1,2, \ldots, m$.

Proof. To prove this, we first try to find out the maximum count of $\alpha$-SEs of $G$. Let $|V|=n$. We know that if more than one $\alpha$-SEs are connected with a common node then the node is a $m \mathrm{PF}$ cut node. Since, $G$ is an $m \mathrm{PF}$ block, therefore it has no $m \mathrm{PF}$ cut node. Therefore, the maximum count of $\alpha$-SEs of $G$ is $\frac{n}{2}$. Thus, $p_{i} \circ \alpha_{V}(G) \leq \frac{\frac{n}{2}}{n}=0.5, \forall i=1,2, \ldots, m$.

Theorem 4.14. An mPF block $G$ be $\alpha$-saturated then $p_{i} \circ \alpha_{V}(G)=0.5, \forall i=1,2, \ldots, m$.

Proof. Let $G$ be $\alpha$-saturated. Since, $G$ is an $m \mathrm{PF}$ block, therefore it has no $m \mathrm{PF}$ cut vertex. Hence, every vertex incident with exactly unique $\alpha$-SE. Therefore, $G$ contains exactly $\frac{n}{2}$ count of $\alpha$-SEs. Thus, $p_{i} \circ \alpha_{V}(G)=\frac{\frac{n}{2}}{n}=0.5$, for $i=1,2, \ldots, m$. 


\section{$5 \quad$ Algorithms}

From Dijkstra's algorithm [5], we first trace $G^{*}=(V, E)$, where $V$ as well as $E$ indicates the set of all nodes as well edges where $|V|=n$.
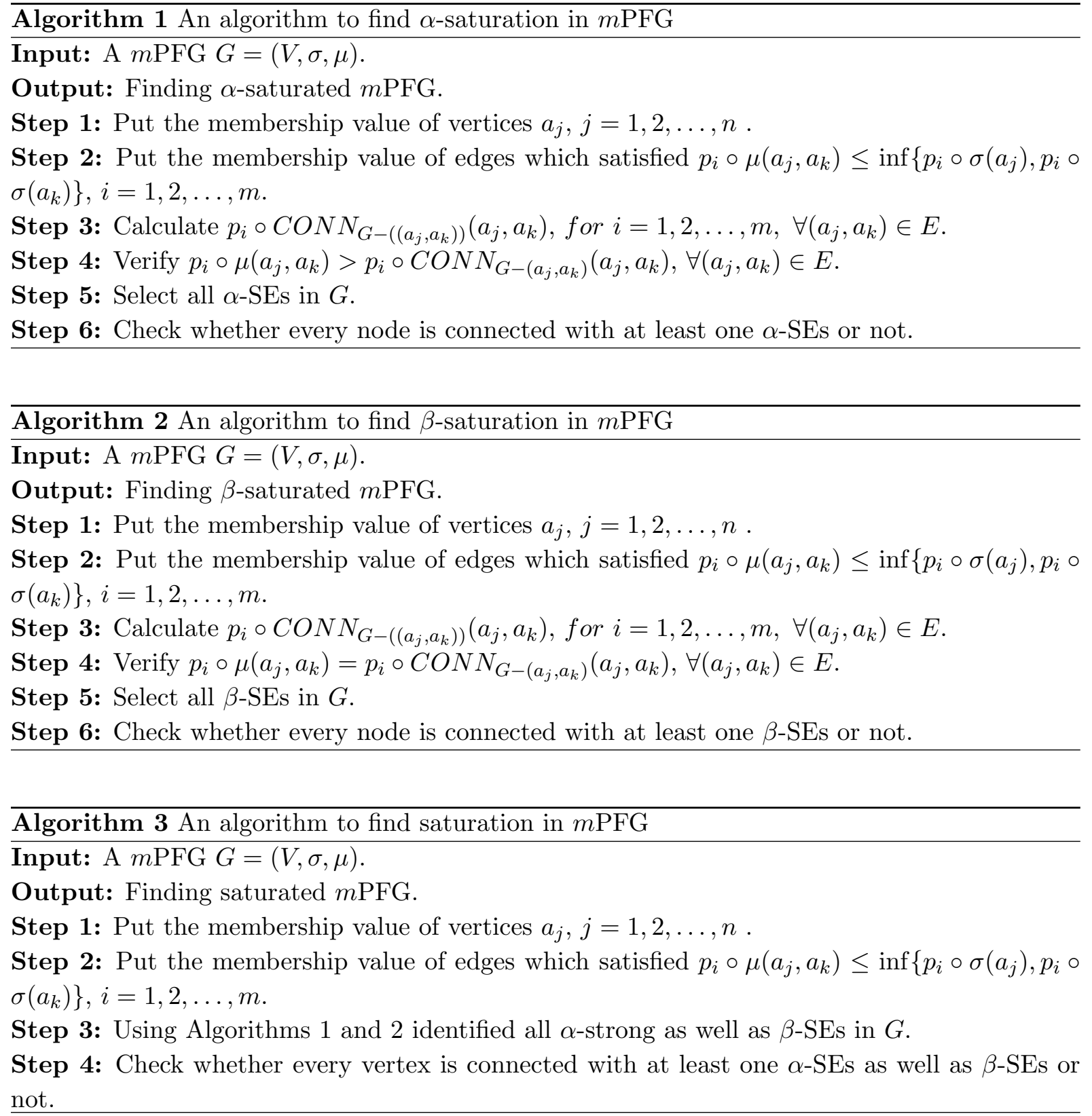


\section{Application}

The $m \mathrm{PFG}$ is an essential mathematical structure representing the facts in real-life connected through graphical systems, in which nodes and edges lie in an $m$-polar fuzzy information. In this section, by using saturation in $m \mathrm{PFG}$, we solve one particular allocation problem.

\subsection{Model construction}

In modern days, education is an essential topic for every person. Under the rule of the Right to Education (RTE) in 2005, everybody has the opportunity to read and write. In the education system, IIT(Indian Institute of Technology) is one of the most important institutions for higher studies in India. Therefore, establishing an IIT in a town among some towns is not an easy task for any Government.

Here, we consider nine towns $a_{1}, a_{2}, \ldots, a_{9}$ as nodes. There will be an edge between two nodes if there exists road connection in between two nodes. Here, we use saturation in 3PFG $G=(V, \sigma, \mu)$ to solve the allocation problem. Since, the towns are fixed in nature therefore we can assign the membership value of each node $(1,1,1)$, that is $\sigma\left(a_{i}\right)=(1,1,1)$, for $i=1,2, \ldots, 9$. The edge membership value is calculated depending on three criteria. Those criteria are as follows: \{Condition of roads, traffic jams on the roads, communication system between two cities\}. All the indicators of an edge between two towns are uncertain in nature. We can calculate the edge membership values by remembering the relation $p_{i} \circ \mu(a, c) \leq \inf \left\{p_{i} \circ \sigma(a), p_{i} \circ \sigma(c)\right\}$, $\forall i=1,2, \ldots, m$. The model $3 \mathrm{PFG}$ is shown in Fig. 5. Here, the edge membership value is

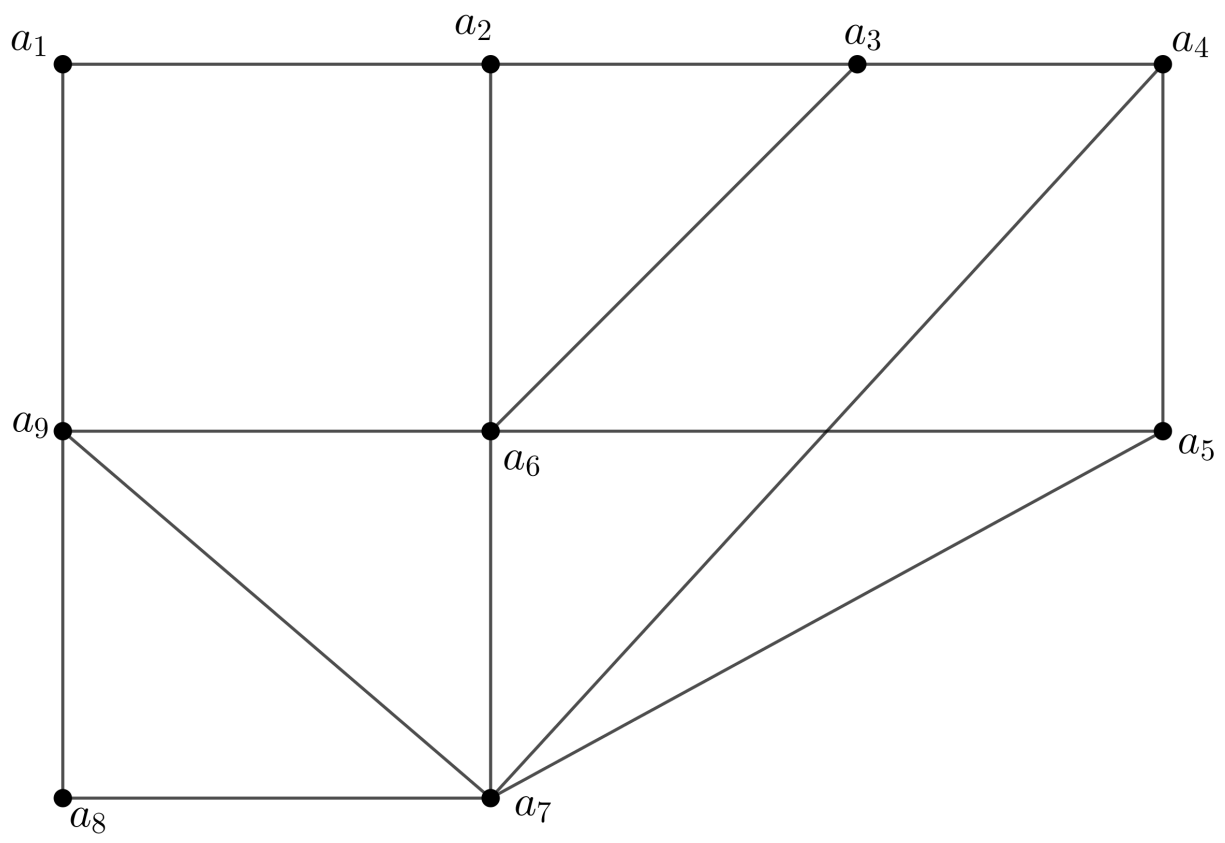

Figure 5: Model 3PFG G 
given in tabular form.

\begin{tabular}{|c|c|}
\hline Edge & Membership Value \\
\hline$\left(a_{1}, a_{2}\right)$ & $(0.5,0.4,0.3)$ \\
\hline$\left(a_{2}, a_{3}\right)$ & $(0.5,0.4,0.3)$ \\
\hline$\left(a_{3}, a_{4}\right)$ & $(0.3,0.2,0.1)$ \\
\hline$\left(a_{4}, a_{5}\right)$ & $(0.3,0.2,0.1)$ \\
\hline$\left(a_{5}, a_{7}\right)$ & $(0.6,0.5,0.4)$ \\
\hline$\left(a_{7}, a_{8}\right)$ & $(0.5,0.4,0.3)$ \\
\hline$\left(a_{8}, a_{9}\right)$ & $(0.3,0.2,0.1)$ \\
\hline$\left(a_{1}, a_{9}\right)$ & $(0.7,0.6,0.5)$ \\
\hline$\left(a_{7}, a_{9}\right)$ & $(0.6,0.5,0.4)$ \\
\hline$\left(a_{6}, a_{9}\right)$ & $(1,0.9,0.8)$ \\
\hline$\left(a_{6}, a_{7}\right)$ & $(0.6,0.5,0.4)$ \\
\hline$\left(a_{5}, a_{6}\right)$ & $(0.6,0.5,0.4)$ \\
\hline$\left(a_{2}, a_{6}\right)$ & $(0.8,0.7,0.6)$ \\
\hline$\left(a_{3}, a_{6}\right)$ & $(0.5,0.4,0.3)$ \\
\hline$\left(a_{4}, a_{7}\right)$ & $(0.7,0.6,0.5)$ \\
\hline
\end{tabular}

\subsection{Illustration of membership values}

Here, the model network system contains nine nodes and fifteen edges. It can be seen from the given 3PFG that every town is connected to others through some paths. So, first, we want to check whether the connections between towns are $\alpha$-strong, $\beta$-strong or $\delta$-strong. Next, we find out the saturation vertex in Fig. 5. After calculating $C O N N_{G-(a, b)}$, for all $(a, b) \in E$, where $E$ is the set of edges of the model $3 \mathrm{PFG}$, we find out which edges are $\alpha$-strong, $\beta$-strong or $\delta$-strong. Then, by the routine computations, we get the classification of edges. Here, the classified edges are given in tabular form. 


\begin{tabular}{|c|r|}
\hline Edge & Classification \\
\hline$\left(a_{1}, a_{2}\right)$ & $\delta$-strong \\
\hline$\left(a_{2}, a_{3}\right)$ & $\delta$-strong \\
\hline$\left(a_{3}, a_{4}\right)$ & $\delta$-strong \\
\hline$\left(a_{4}, a_{5}\right)$ & $\delta$-strong \\
\hline$\left(a_{5}, a_{7}\right)$ & $\delta$-strong \\
\hline$\left(a_{7}, a_{8}\right)$ & $\alpha$-strong \\
\hline$\left(a_{8}, a_{9}\right)$ & $\delta$-strong \\
\hline$\left(a_{1}, a_{9}\right)$ & $\alpha$-strong \\
\hline$\left(a_{7}, a_{9}\right)$ & $\delta$-strong \\
\hline$\left(a_{6}, a_{9}\right)$ & $\alpha$-strong \\
\hline$\left(a_{6}, a_{7}\right)$ & $\delta$-strong \\
\hline$\left(a_{5}, a_{6}\right)$ & $\delta$-strong \\
\hline$\left(a_{2}, a_{6}\right)$ & $\alpha$-strong \\
\hline$\left(a_{3}, a_{6}\right)$ & $\beta$-strong \\
\hline$\left(a_{4}, a_{7}\right)$ & $\alpha$-strong \\
\hline
\end{tabular}

Here, only one $\beta$-SEs are present in the model 3PFG $G$. The node $a_{6}$ is the only saturation node in the model 3PFG $G$ as it is an incident with at least one $\alpha$-SE and one $\beta$-SEs.

\subsection{Decision making}

Since $a_{6}$ is the only saturation node in the model 3 PFG $G$, we can say that the town $a_{6}$ is the most suitable place to establish the IIT (Indian Institute of Technology) among all other towns considered in this proposed model.

We know that saturation in $m \mathrm{PFG}$ plays an essential role in this type of allocation problem through the above discussion. Moreover, we also recognize that saturation in $m \mathrm{PFG}$ is more applicable than saturation in FG in allocation problems.

\section{Conclusion}

In this paper, $\alpha$-saturation as well as $\beta$-saturation in $m \mathrm{PFG}$ along with its several properties are initiated. Vertex and edge saturation count in $m \mathrm{PFG}$ and a few of its facts on some well known $m$ PFG is also introduced. The upper and lower bound of a vertex as well as edge saturation count in $m \mathrm{PFG}$ are also investigated. Saturation in $m \mathrm{PFG}$ by using $\alpha$-saturation as well as $\beta$ saturation are also discussed here along with some of its intersecting properties. Using saturation in $m \mathrm{PFG}$, an application is also given in the last part of this paper. Our research work will be extended depending on $m \mathrm{PFG}$ to find more characteristics and applications. 


\section{Compliance with ethical standards}

Ethical approval This article does not contain any studies with human participants or animals performed by any of the authors.

Conflict of interest It has been declared by the authors that no conflict of interest of any person(s) or organization(s) has happened.

Funding details This research has no funding by any organization or individual.

Informed Consent It was obtained from all individual participants included in the study.

\section{Authors' contributions}

All the authors contribute equally in this work.

\section{References}

[1] Akram M and Adeel A (2017) $m$-polar fuzzy graphs and $m$-polar fuzzy line graphs. Journal of Discrete Mathematical Sciences and Cryptography 20(8): 1597-1617.

[2] Akram M, Wassem N and Dudek W A (2016) Certain types of edge $m$-polar fuzzy graph. Iranian Journal of Fuzzy System 14(4): 27-50.

[3] Akram M (2019) m-polar fuzzy graphs, theory, methods, application. DOI: 10.1007/978-3030-03751-2, Springer International Publishing.

[4] Chen J, Li S, Ma S, and Wang X (2014) m-polar fuzzy sets: an extension of bipolar fuzzy sets. The Scientific World Journal 2014: 1-8.

[5] Douglas B W (2002) Introduction to graph theory. Pearson Education India, Noida

[6] Ghorai G and Pal M (2015) On some operations and density of $m$-polar fuzzy graphs. Pacific Science Review A: Natural Science and Engineering 17(1): 14-22.

[7] Ghorai G and Pal M (2016) Some properties of $m$-polar fuzzy graphs. Pacific Science Review A: Natural Science and Engineering 18: 38-46.

[8] Ghorai G and Pal M (2016) A study on $m$-polar fuzzy planar graphs. International Journal of Computing Science and Mathematics 7(3): 283-292.

[9] Ghorai G and Pal M (2016) Faces and dual of $m$-polar fuzzy planar graphs. Journal of Intelligent and Fuzzy Systems 31: 2043-2049.

[10] Kauffman A (1973) Introduction a la Theorie des Sous-emsembles Flous, Mansson et Cie 1: 1973.

[11] Mahapatra T and Pal M (2018) Fuzzy colouring of $m$-polar fuzzy graph and its application. Journal of Intelligent and Fuzzy Systems 35(6): 6379-6391. 
[12] Mahapatra T, Ghorai G and Pal M (2020) Fuzzy fractional coloring on fuzzy graph with its application. Journal of Ambient Intelligence and Humanized Computing https://doi.org/10.1007/s12652-020-01953-9.

[13] Mandal S, Sahoo S, Ghorai G and Pal M (2018) Different Types of Arcs in $m$-polar Fuzzy Graphs with Application. J. of Mult. Valued Logic and Soft Computing 34: 263-282.

[14] Mandal S, Sahoo S, Ghorai G and Pal M (2018) Application of SEs in m-Polar Fuzzy Graphs. Neural Processing Letters https://doi.org/10.1007/s11063-018-9934-1.

[15] Mathew S and Sunitha M S (2012) Fuzzy graphs: basics, concepts and applications. Lap Lambert Academic Publishing.

[16] Mathew S, Yang L H and Mathew K J (2018) Saturation in Fuzzy Graphs New Mathematics and Natural Computation 14(1): 113-128.

[17] Mordeson J N and Nair P S (2000) Fuzzy graph and fuzzy hypergraphs. Physica-Verlag Heidelberg.

[18] Nair P S and Cheng S C (2001) Cliques and fuzzy cliques in fuzzy graphs. IFSA World Congress and 20th NAFIPS International Conference 4: 2277-2280.

[19] Pal M, Samanta S, Ghorai G (2020) Modern Trends in Fuzzy Graph Theory, Springer.

[20] Rosenfeld A (1975) Fuzzy Graphs, fuzzy sets and their application. Academic Press,New York. pp. 77-95.

[21] Sunitha S M and Mathew S (2013) Fuzzy graph theory: a survey. Annals of Pure and Applied Mathematics 4: 92-110.

[22] Zhang R W (1994) Bipolar fuzzy sets and relations:a computational framework for cognitive modeling and multiagent decision analysis, Proceedings of IEEE Conference 305-309.

[23] Zhang R W (1998) Bipolar fuzzy sets. Proceedings of IEEE Conference 835-840.

[24] Zadeh L A (1965) Fuzzy sets. Information and Control: 338-353. 